Article

\title{
Genomics of Methylotrophy in Gram-Positive Methylamine-Utilizing Bacteria
}

Tami L. McTaggart ${ }^{1, \dagger}$, David A. C. Beck ${ }^{1,3}$, Usanisa Setboonsarng ${ }^{1, \star}$, Nicole Shapiro ${ }^{4}$, Tanja Woyke ${ }^{4}$, Mary E. Lidstrom ${ }^{1,2}$, Marina G. Kalyuzhnaya ${ }^{2, \S}$ and Ludmila Chistoserdova ${ }^{1, *}$

1 Department of Chemical Engineering, University of Washington, Seattle, WA 98195, USA; E-Mails: tmctagga@uci.edu (T.L.M.); dacb@uw.edu (D.A.C.B.); lisa.setboonsarng@gmail.com (U.S.); lidstrom@uw.edu (M.E.L.)

2 Department of Microbiology, University of Washington, Seattle, WA 98195, USA; E-Mail: mkalyuzhnaya@mail.sdsu.edu

3 eScience Institute, University of Washington, Seattle, WA 98195, USA

4 DOE Joint Genome Institute, Walnut Creek, CA 94598, USA; E-Mails: nrshapiro@1bl.gov (N.S.); TWoyke@lbl.gov (T.W.)

$\dagger$ Present address: Department of Chemical Engineering and Materials Science, University of California Irvine, Irvine, CA 92697, USA.

Present address: Denali Advanced Integration, Redmond, WA 98052, USA.

$\S$ Present address: Biology Department, San Diego State University, San Diego, CA 92182, USA.

* Author to whom correspondence should be addressed; E-Mail: milachis@u.washington.edu; Tel.: +1-206-616-1913.

Academic Editor: Andrew McDowell

Received: 3 February 2015 / Accepted: 6 March 2015 / Published: 20 March 2015

\begin{abstract}
Gram-positive methylotrophic bacteria have been known for a long period of time, some serving as model organisms for characterizing the specific details of methylotrophy pathways/enzymes within this group. However, genome-based knowledge of methylotrophy within this group has been so far limited to a single species, Bacillus methanolicus (Firmicutes). The paucity of whole-genome data for Gram-positive methylotrophs limits our global understanding of methylotrophy within this group, including their roles in specific biogeochemical cycles, as well as their biotechnological potential. Here, we describe the isolation of seven novel strains of Gram-positive
\end{abstract}


methylotrophs that include two strains of Bacillus and five representatives of Actinobacteria classified within two genera, Arthrobacter and Mycobacterium. We report whole-genome sequences for these isolates and present comparative analysis of the methylotrophy functional modules within these genomes. The genomic sequences of these seven novel organisms, all capable of growth on methylated amines, present an important reference dataset for understanding the genomic basis of methylotrophy in Gram-positive methylotrophic bacteria. This study is a major contribution to the field of methylotrophy, aimed at closing the gap in the genomic knowledge of methylotrophy within this diverse group of bacteria.

Keywords: methylotrophy; genomics; Arthrobacter; Bacillus; Mycobacterium; Lake Washington

\section{Introduction}

Methylotrophy, the ability to utilize substrates containing no carbon-carbon bonds (C1 substrates), is widespread in the domain of Bacteria, with representatives found in Proteobacteria, Firmicutes, Actinobacteria, Verrucomicrobia and the NC10 candidate phylum [1,2]. At the genomic level, methylotrophy has been characterized in detail in Proteobacteria, with the first genome published in 2004 [3] and the count of currently available genomes nearing one hundred [2]. While methylotroph representatives within Verrucomicrobia and the $\mathrm{NC10}$ candidate phylum have only been characterized recently, representative genome sequences are already available [4-6].

Gram-positive methylotrophs have been known for a long time, some serving as model organisms for characterizing the specific details of methylotrophy pathways/enzymes within this group [1,7-12]. However, whole-genome knowledge of methylotrophy in Gram-positive methylotrophs has been limited to a single species, Bacillus methanolicus, within the Firmicutes [13-15]. Methylotrophy pathways have been analyzed in this organism at the systems level [15]. In addition, a large body of research exists on individual enzymes/pathways in a variety of Gram-positive methylotrophs, providing helpful clues as to the important methylotrophy activities in these organisms [1,7-12,15-18]. Based on the prior research, the methylotrophy metabolic scheme in Gram-positive bacteria is as follows. Primary C1 substrates, methanol or methylated amines, are oxidized to produce formaldehyde, which is assimilated via the ribulose monophosphate (RuMP) cycle into biomass (fructose 1,6-bisphosphate aldolase variant [1,15]. The oxidative branch of the RuMP cycle serves to oxidize formaldehyde to $\mathrm{CO}_{2}$. However, a linear route with formate as an intermediate may also be functional. In B. methanolicus, the linear route involves tetrahydrofolate $(\mathrm{H} 4 \mathrm{~F})$-linked $\mathrm{C} 1$ transfer reactions [15].

The goal of this study was to expand the whole-genome knowledge of methylotrophy in Gram-positive bacteria through comparative genomics of divergent taxa within this group. Here, we describe the isolation of seven novel strains of Gram-positive methylotrophs, including two strains of Bacillus (Bacillaceae) and five representatives of Actinobacteria, belonging to the genera of Arthrobacter (Micrococcaceae) and Mycobacterium (Microbacteriaceae). We report whole-genome sequences for 
these isolates and present a comparative analysis of methylotrophy functional modules within these genomes.

\section{Materials and Methods}

\subsection{Permissions}

No specific permissions were required for the sampling location $\left(47^{\circ} 38.075^{\prime} \mathrm{N}, 122^{\circ} 15.993^{\prime} \mathrm{W}\right)$ or sampling activities. The field studies did not involve endangered or protected species.

\subsection{Strain Isolation and Cultivation}

Arthrobacter sp. MA-N2 was isolated from a 2004 Lake Washington sample [19], and the rest of the strains were isolated from a 2011 Lake Washington sample ([20]; Table 1). All strains were isolated from methylamine enrichments, which were set up as previously described [20,21]. Axenic cultures were routinely maintained on solid media supplemented by methylamine. For long-term storage, culture stocks were frozen at $-80^{\circ}$ with $10 \%$ dimethyl sulfoxide, as a cryoprotective agent.

\subsection{DNA Isolation, Whole Genome Sequencing, Assembly and Genome Annotation}

Biomass for genomic DNA isolation was collected from plates. DNA was isolated as previously described [22]. The draft genomes were generated at the Department of Energy Joint genome Institute (JGI) using Illumina, Pacific Biosciences (PacBio) or a combination of the two technologies (see Table 1). All general aspects of library construction and sequencing performed at the JGI can be found at http://www.jgi.doe.gov. The raw reads were assembled using HGAP (version: 2.0.0) [23] for PacBio datasets, Allpaths, Version r41554, for hybrid Illumina/PacBio datasets, and a combination of Allpaths, Version r41554 [24], and Velvet, Version 1.1.05 [25], for the Illumina datasets. Genes were identified using Prodigal [26], followed by a round of manual curation using GenePRIMP [27] for the Draft genomes in fewer than 10 scaffolds. The predicted coding sequences were translated and used to search the National Center for Biotechnology Information (NCBI) non-redundant database, Universal Protein Resource (UniProt), TIGRFam, Pfam, Kyoto Encyclopedia of Genes and Genomes (KEGG), COG, and InterPro databases. The tRNAScanSE tool [28] was used to find tRNA genes, whereas ribosomal RNA genes were found by searches against models of the ribosomal RNA genes built from SILVA [29]. Other non-coding RNAs, such as the RNA components of the protein secretion complex and the RNase P, were identified by searching the genome for the corresponding Rfam profiles using INFERence of RNA Alignment (INFERNAL) [30]. Additional gene prediction analysis and manual functional annotation was performed within the Integrated Microbial Genomes (IMG) platform developed by the Joint Genome Institute, Walnut Creek, CA, USA [31].

The genomes are accessible through the IMG interface (http://img.jgi.doe.gov/cgi-bin/w/main.cgi). 
Table 1. Strain isolation details, genome statistics and accession numbers.

\begin{tabular}{ccccccccc}
\hline Strain & Year Isolated & $\begin{array}{c}\text { Enrichment } \\
\text { Temperature }{ }^{\circ} \mathbf{C}\end{array}$ & Total Nucleotides & GC\% & $\begin{array}{c}\text { Sequencing } \\
\text { Technology }\end{array}$ & Scaffolds & $\begin{array}{c}\text { Coverage } \\
\text { (X) }\end{array}$ & $\begin{array}{c}\text { NCBI Accession } \\
\text { Number }\end{array}$ \\
\hline Arthrobacter sp. 31Y & 2011 & 10 & $5,079,550$ & 61.94 & PacBio & 2 & 140 & JAFW00000000.1 \\
Arthrobacter sp. 35W & 2011 & 10 & $4,660,196$ & 66.71 & Illumina/PacBio & 4 & $1647 / 130$ & AXVQ00000000.1 \\
Arthrobacter sp. MA-N2 & 2004 & Room & $4,833,792$ & 62.96 & Illumina & 5 & 793 & AQRI010000000.1 \\
Bacillus sp. 37MA & 2011 & 10 & $3,981,584$ & 40.52 & Illumina & 5 & 1091 & ARCN01000000.1 \\
Bacillus sp. 72 & 2011 & 10 & $3,908,751$ & 40.85 & PacBio & 26 & 180 & JQMI01000000.1 \\
Mycobacterium sp. 141 & 2011 & 30 & $4,544,736$ & 65.57 & Illumina & 3 & 1171 & ARNS01000000.1 \\
Mycobacterium sp. 155 & 2011 & 30 & $4,609,894$ & 65.62 & Illumina & 2 & 1451 & AREU01000000.1 \\
\hline
\end{tabular}




\subsection{Phylogenetic Analysis}

Average amino acid identity (AAI) values were computed via reciprocal BLAST best hits between pairs of proteomes in accordance with previously described methods [32], except that the predicted protein products were used directly as the subject for alignments rather than translated genomic sequences [20,21].

\subsection{Reconstruction of Methylotrophy Pathways}

Automated gene annotations created using the IMG pipeline were curated manually for genes involved in key metabolic pathways. Reconstruction of methylotrophy pathways was modeled after prior analysis of the genomes of B. methanolicus [13-15]. Proteins with experimentally proven functions in methylated amine metabolism were also employed [10,18]. In the case of multiple functional counterparts, these were categorized into "types" based on reciprocal BLAST comparisons. In the cases of very divergent counterparts, some of the "type" categories are very tentative, as low homology may result either from a long evolutionary history or from a lateral transfer from a distant source.

\section{Results and Discussion}

\subsection{Gram-Positive Methylotrophs Isolated from Lake Washington Are All Facultative Methylated Amine Utilizers}

As part of a large-scale methylotroph isolation project from Lake Washington sediment samples, a number of Gram-positive strains were isolated. Three of the isolates described here were identified as Arthrobacter, two as Bacillus and two as Mycobacterium species (Table 1). These were selected for genomic sequencing out of multiple isolates, and each isolate in the same phylogenetic affiliation originated from a different enrichment microcosm. The Arthrobacter and the Bacillus strains were only isolated from the enrichments set up at $10{ }^{\circ} \mathrm{C}$ or at room temperature, while the Mycobacterium strains were only isolated from enrichments set up at $30{ }^{\circ} \mathrm{C}$. The Arthrobacter species were represented by two distinct phylotypes, forming either bright yellow (strain 31Y) or whitish colonies (strains 35W and MA-N2). The two Bacillus isolates and the two Mycobacterium isolates, respectively, were similar phenotypically. All isolates were facultative methylotrophs, able to grow on rich media, such as nutrient broth agar (Difco), and on multiple multicarbon substrates, such as succinate or glucose, as previously reported for these types [1]. However, none was able to grow on methanol.

\subsection{The Newly-Sequenced Genomes Represent a Diversity of Gram-Positive Methylotrophs}

Based on the 16S rRNA gene sequence, the closest named relative of all three Arthrobacter isolates was Arthrobacter aurescens (97.4\% to $99.8 \%$ 16S rRNA gene sequence identity). However, they were only distantly related to each other $(96.7 \%$ to $97.5 \% 16 \mathrm{~S}$ rRNA gene sequence identity). Both white isolates were somewhat more closely related to the yellow isolate than to each other.

The two Mycobacterium isolates were closely related to each other (99.4\%) and closely related to the differently named species of Mycobacterium (M. rhodesiae, M. gilvum, M. smegmatis; 97.6\% to $97.8 \%$ ). 
The two Bacillus isolates were closely related to each other $(100 \% 16 \mathrm{~S}$ rRNA gene sequence identity), but not to the described Bacillus species, including the well-studied B. methanolicus species strains, MGA3 and PB1, with which they only showed 93.0\% and 93.1\% 16S rRNA gene identity, respectively.

We further assessed the genomic diversity of the isolates in terms of genome-genome similarity, via calculating average amino acid identity indices (AAI) [16,17]. As expected, the two Mycobacterium genomes and the two Bacillus genomes were found to be very similar in terms of protein-protein identity (95\% and 98\% AAI, respectively; Figure 1). However, the proteins translated from the Bacillus genomes shared only $61.2 \%$ AAI with the proteome of B. methanolicus. The Bacillus (low GC Gram-positive organisms) proteomes shared only 39\% AAI with the Arthrobacter or the Mycobacterium (high GC Gram-positive organisms) proteomes. Within the high GC Gram-positive group, significant divergence was uncovered, with the three Arthrobacter species only sharing $68 \%$ to 78\% AAI and the Mycobacterium genomes sharing only 50\% AAI with the Arthrobacter genomes (Figure 1).

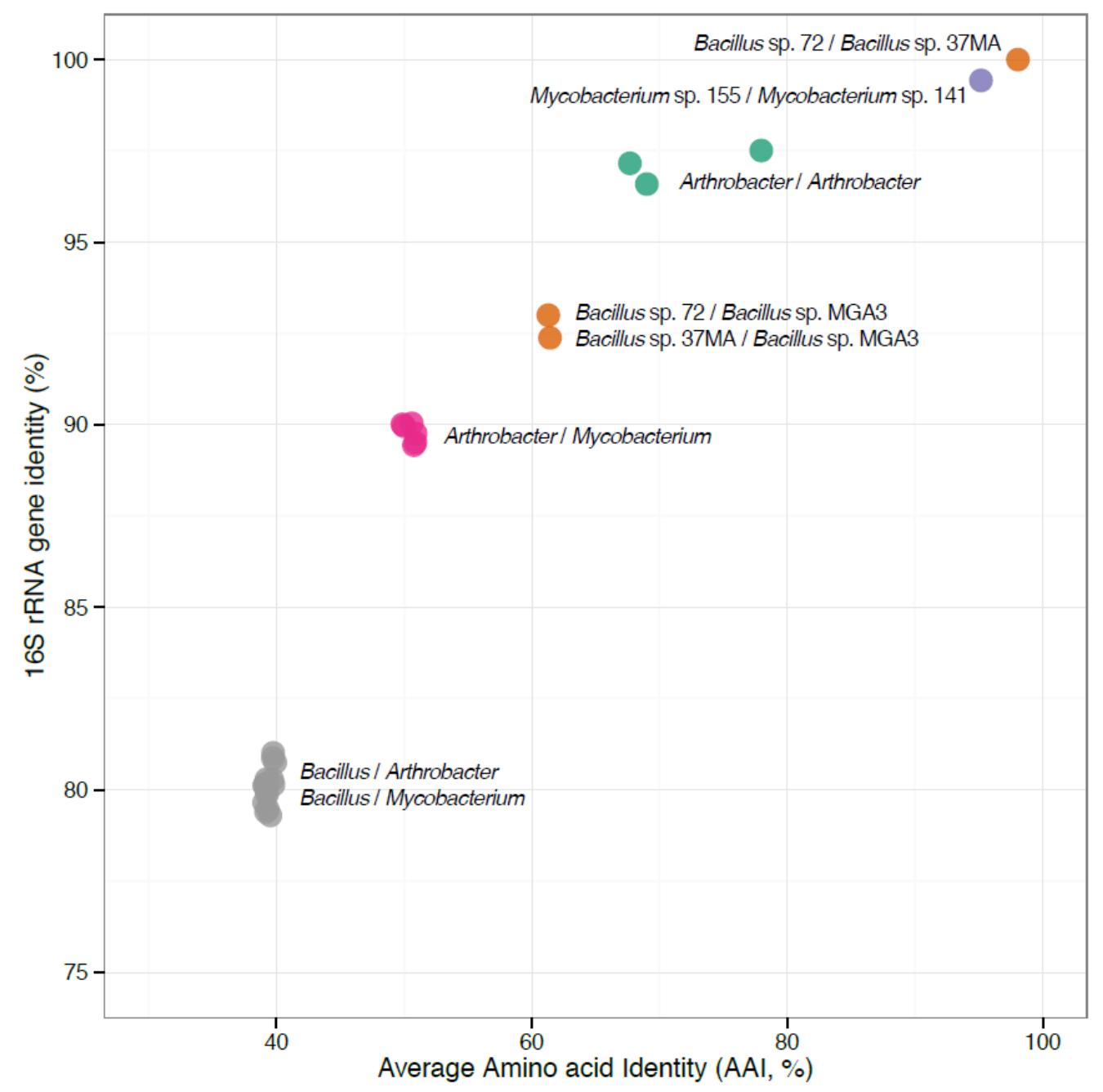

Figure 1. Phylogenetic relationships among the strains described. Each dot represents a comparison between two genomes and shows their 16S rRNA gene identity ( $y$-axis) plotted against the average amino acid identity (AAI) of the genes shared between the two genomes ( $x$-axis). 


\subsection{Methylotrophy Pathways Deduced from the Novel Genomes Agree with Prior Knowledge, but} Suggest Novel Primary Oxidation Modules

Methylotrophy pathways so far have been only thoroughly analyzed at the systems level in one representative of Gram-positive methylotrophs, B. methanolicus. The methylotrophic metabolic scheme in this bacterium [13-15], as well as the individual methylotrophy genes previously identified via functional studies in a variety of Gram-positive methylotrophs $[9,10,12,18]$ served as guides in reconstructing methylotrophy metabolic modules in the new genomes.

\subsubsection{Primary C1 Oxidation Modules}

None of the organisms included in this study were able to grow on methanol. Thus, not surprisingly, no genes for methanol oxidation were identified in any of the genomes, by using protein queries for the NAD-dependent methanol dehydrogenase from B. methanolicus [13,15] or the $\mathrm{N}, \mathrm{N}$-dimethyl-4-nitrosoamiline oxidoreductase from Mycobacterium sp. [12].

Queries for methylated amine oxidation modules produced the following results. Genes for trimethylamine monooxygenase (Tmo) [18] were identifiable in the genomes of Arthrobacter (two copies in strain MA-N2) and Mycobacterium strains, but not in the Bacillus genomes (Table 2). The Arthrobacter counterparts shared approximately $90 \%$ amino acid identities (AI), while Arthrobacter/Mycobacterium AI values were approximately $87 \%$, which is significantly above the AAI values (Figure 1). The identities with proteobacterial counterparts, including the characterized proteins from alphaproteobacteria [18], were approximately $60 \%$. Likely, the same proteins are responsible for the dimethylamine monooxygenase activity, as no additional candidates for this activity were identifiable.

Genes encoding methylamine oxidases (Mao) were identified via comparisons with the sequence of the biochemically characterized Mao from Arthrobacter P1 [10,11]. Arthrobacter strains 31Y and $35 \mathrm{~W}$ each encoded two nearly-identical copies, both parts of an extended cluster encoding other methylotrophy functions (Figure 2). In the genome of strain MA-N2, two full-length gene copies are identifiable and a number of partial genes. As this is a draft sequence, some of the incomplete copies may be the result of poor assembly. Alternatively, they may be the result of genomic evolution. The Bacillus genomes encode three nearly-identical copies each, with approximately AI $45 \%$ with the Arthrobacter counterparts. 
Table 2. Enzymes involved in methylotrophy.

\begin{tabular}{|c|c|c|c|c|c|c|c|}
\hline & $\begin{array}{l}\text { Arthrobacter sp. } \\
\text { 31Y }\end{array}$ & $\begin{array}{c}\text { Arthrobacter sp. } \\
\text { 35W }\end{array}$ & $\begin{array}{c}\text { Arthrobacter sp. } \\
\text { M-N2 }\end{array}$ & $\begin{array}{c}\text { Bacillus sp. } \\
\text { 37MA }\end{array}$ & $\begin{array}{c}\text { Bacillus sp. } \\
72\end{array}$ & $\begin{array}{c}\text { Mycobacterium sp. } \\
141\end{array}$ & $\begin{array}{c}\text { Mycobacterium sp. } \\
155\end{array}$ \\
\hline Tmo & 1611 & 3598 & 1313,1316 & - & - & 2303 & 3391 \\
\hline Tmd & 2725 & 3579 & 1325 & - & - & 2887 & 3922 \\
\hline Mao & 2723,2743 & 3577,3611 & $\begin{array}{c}1074,1322 / 1323,3581,4240 \\
4249\end{array}$ & $\begin{array}{l}1646,4043 \\
4264 / 4265\end{array}$ & $\begin{array}{c}0038,0528 \\
3848\end{array}$ & 2885 (partial) & 3920 (partial) \\
\hline EutQ * & $\begin{array}{c}1610,1786,2727, \\
2952\end{array}$ & $1944,3581,3597$ & $1314,1315,1327$ & $\begin{array}{c}2690,2768,4276 \\
4302\end{array}$ & 0506,0538 & 2304,2889 & $1719,3392,3929$ \\
\hline Gma & - & - & - & - & - & 2874 & 3910 \\
\hline MgsA & - & - & - & - & - & 2875 & 3911 \\
\hline MgsB & - & - & - & - & - & 2876 & 3912 \\
\hline $\mathrm{MgsC}$ & - & - & - & - & - & 2877 & 3913 \\
\hline $\operatorname{MgdA}$ & 2010 & - & 1962 & - & - & 1509 & 2645 \\
\hline MgdB & 2011 & - & 1963 & - & - & 1510 & 2646 \\
\hline MgdC & 2012 & - & 1964 & - & - & 1511 & 2647 \\
\hline MgdD & 2013 & - & 1965 & - & - & 1512 & 2648 \\
\hline FolD1 & 3659 & 0111 & 2114 & 2264 & 1211 & 1451 & 2593 \\
\hline FolD2 & 2859 & - & - & - & - & - & - \\
\hline Mch & 2860 & - & - & - & - & - & - \\
\hline PurU1 & 3656 & 0108 & 2111 & 3324 & 2413 & 1098,2609 & 2235,3667 \\
\hline PurU2 & 2015 & - & 1967 & - & - & - & - \\
\hline PurU3 & 2862 & - & - & - & - & - & - \\
\hline Fhs & 0972 & - & 0487 & - & - & - & - \\
\hline $\mathrm{FaDH}$ & 2857 & - & - & - & - & 2016 & - \\
\hline Fdh1A & - & - & 3202 & - & - & 1890 & $3030 / 3031$ \\
\hline Fdh1B & - & - & 3201 & - & - & 1889 & 3029 \\
\hline
\end{tabular}


Table 2. Cont.

\begin{tabular}{|c|c|c|c|c|c|c|c|}
\hline & $\begin{array}{c}\text { Arthrobacter sp. } \\
\text { 31Y }\end{array}$ & $\begin{array}{c}\text { Arthrobacter sp. } \\
\text { 35W }\end{array}$ & $\begin{array}{c}\text { Arthrobacter sp. } \\
\text { M-N2 }\end{array}$ & $\begin{array}{c}\text { Bacillu sp. } \\
\text { 37MA }\end{array}$ & $\begin{array}{c}\text { Bacillus sp. } \\
72\end{array}$ & $\begin{array}{c}\text { Mycobacterium sp. } \\
141\end{array}$ & $\begin{array}{c}\text { Mycobacterium sp. } \\
155\end{array}$ \\
\hline $\mathrm{dh} 1 \mathrm{C}$ & - & - & 3200 & - & - & 1888 & 3028 \\
\hline Fdh2 & - & - & - & 3013 & 1944 & - & - \\
\hline Fdh3 & 3415 & - & 1898 & - & - & - & - \\
\hline Fdh4 & 2858 & 0024 & - & - & - & - & - \\
\hline Fdh5 & - & - & 3418 & - & - & - & - \\
\hline Pgil & 4707 & 2121 & 1329 & - & - & - & - \\
\hline Pgi2 & - & - & - & 2633 & 1591 & - & - \\
\hline Pgi3 & - & - & - & - & - & 1659,1660 & 2797,2798 \\
\hline Zwf1 & 2734,4706 & $1501,3590,-4201$ & 1079,1690 & - & - & 0485 & 1591 \\
\hline Zwf2 & 0544 & 2030 & 0009 & - & - & - & - \\
\hline Zwf3 & - & - & - & - & - & 0102,3386 & 0013 \\
\hline Zwf4 & - & - & - & 2231 & 1177 & - & - \\
\hline Zwf5 & - & - & - & 4315 & 0509 & - & - \\
\hline OpcA * & 2735,4705 & $1502,3591,4202$ & $\begin{array}{c}1078,1330 / 1331,1691,3346 \\
3572 / 3573\end{array}$ & - & - & 0486 & 1592 \\
\hline $\mathrm{Pgl}$ & 4704 & 1503 & 3347,3572 & 0487 & 1593 & 3368,3220 & 2456,2308 \\
\hline Gnd1 & 0504 & 1989,3592 & 1692,3535 & 2234,3220 & 1184,2308 & & \\
\hline Gnd2 & 2377 & 3418 & 1072,4056 & - & - & 0096,2519 & 3586 \\
\hline Gnd3 & - & - & - & 4314 & 0510 & - & - \\
\hline Hps & 2720,2732 & 2137,3588 & $1082,1667,1687$ & $\begin{array}{c}2007,2010,4272 / 4273 \\
4303,4317\end{array}$ & $\begin{array}{c}0507,0521,0535 \\
2219,2222\end{array}$ & 2900 & 3939 \\
\hline Hpi & 2731 & 2136,3587 & $1083,1668,1686$ & 4274 & 0522,0536 & 3940,3946 & 2901, 2907 \\
\hline Pfk1 & 1057,2718 & 2691,3605 & $0573,1061,4242,4265$ & 4309 & 0515 & 2904 & 3943 \\
\hline Pfk2 & - & - & - & 2525 & 1481 & 0976 & 2045 \\
\hline
\end{tabular}


Table 2. Cont.

\begin{tabular}{|c|c|c|c|c|c|c|c|}
\hline & Arthrobacter sp. 31Y & $\begin{array}{c}\text { Arthrobacter sp. } \\
35 \mathrm{~W}\end{array}$ & $\begin{array}{c}\text { Arthrobacter sp. } \\
\text { M-N2 }\end{array}$ & $\begin{array}{c}\text { Bacillus sp. } \\
\text { 37MA }\end{array}$ & $\begin{array}{c}\text { Bacillus sp. } \\
72\end{array}$ & $\begin{array}{c}\text { Mycobacterium sp. } \\
141 \\
\end{array}$ & $\begin{array}{c}\text { Mycobacterium sp. } \\
155 \\
\end{array}$ \\
\hline Pfk3 & 4492 & - & - & - & - & - & - \\
\hline Fba & 2746,2929 & 3604,3822 & 1066,1250 & 3273,4307 & 0517,2360 & 1980,2894 & 3125,3933 \\
\hline Tkt & 2737,4709 & 1498,3600 & $1070,1091,3342$ & 1128,4308 & 0372,0576 & 0483,2898 & 1589,3937 \\
\hline Tal & 2738,4708 & 1499,3601 & $1069,1090,3343$ & 3279 & 2359 & 0484,2897 & 1590,3936 \\
\hline GlpX1 & 3729 & 0180 & 2184 & 3270 & 2355 & 3603 & 0231 \\
\hline GlpX2 & - & - & - & 3354,4306 & 0578,2442 & - & - \\
\hline Rpe & 2739,4222 & 0804,3602 & $1068,1089,2663$ & 4305 & 0519 & 2896 & 3935 \\
\hline Rpi1 & 0371,2740 & 1824,3603 & $1067,1088,3504$ & 4310 & 0514 & 2895 & 3934 \\
\hline Rpi2 & 2802,3510 & 1140 & 0961 & 3357 & 2445 & - & - \\
\hline Tpil & 4701 & 1505 & 3370 & 2917 & 1868 & 0491 & 1597 \\
\hline Tpi2 & 2801 & 1141 & - & - & - & - & - \\
\hline Tpi3 & 3515 & - & - & - & - & - & - \\
\hline Gap1 & 4699 & 1507 & 3352 & 2512,2919 & 1468,1870 & 0493 & 1599 \\
\hline Gap2 & 0418,2719 & 1975,3607 & $1060,3526,4241$ & - & - & - & - \\
\hline Pgk & 4700 & 1506 & 3351 & 2918 & 1869 & 0492 & 1598 \\
\hline Eda & 2236 & - & 4368,4405 & - & - & - & - \\
\hline
\end{tabular}

For enzyme abbreviations, see the text. Numbers correspond to gene numbering in each genome as annotated in the Integrated Microbial Genomes (IMG)/M database (http://img.jgi.doe.gov/cgi-bin/w/main.cgi). * EutQ, protein of unknown function that we propose to be involved in methylated amine oxidation, based on its conspicuous location near genes for Mao, Tmo and Tmd; OpcA, protein essential for the activity of Zwf in some organisms [33]. 

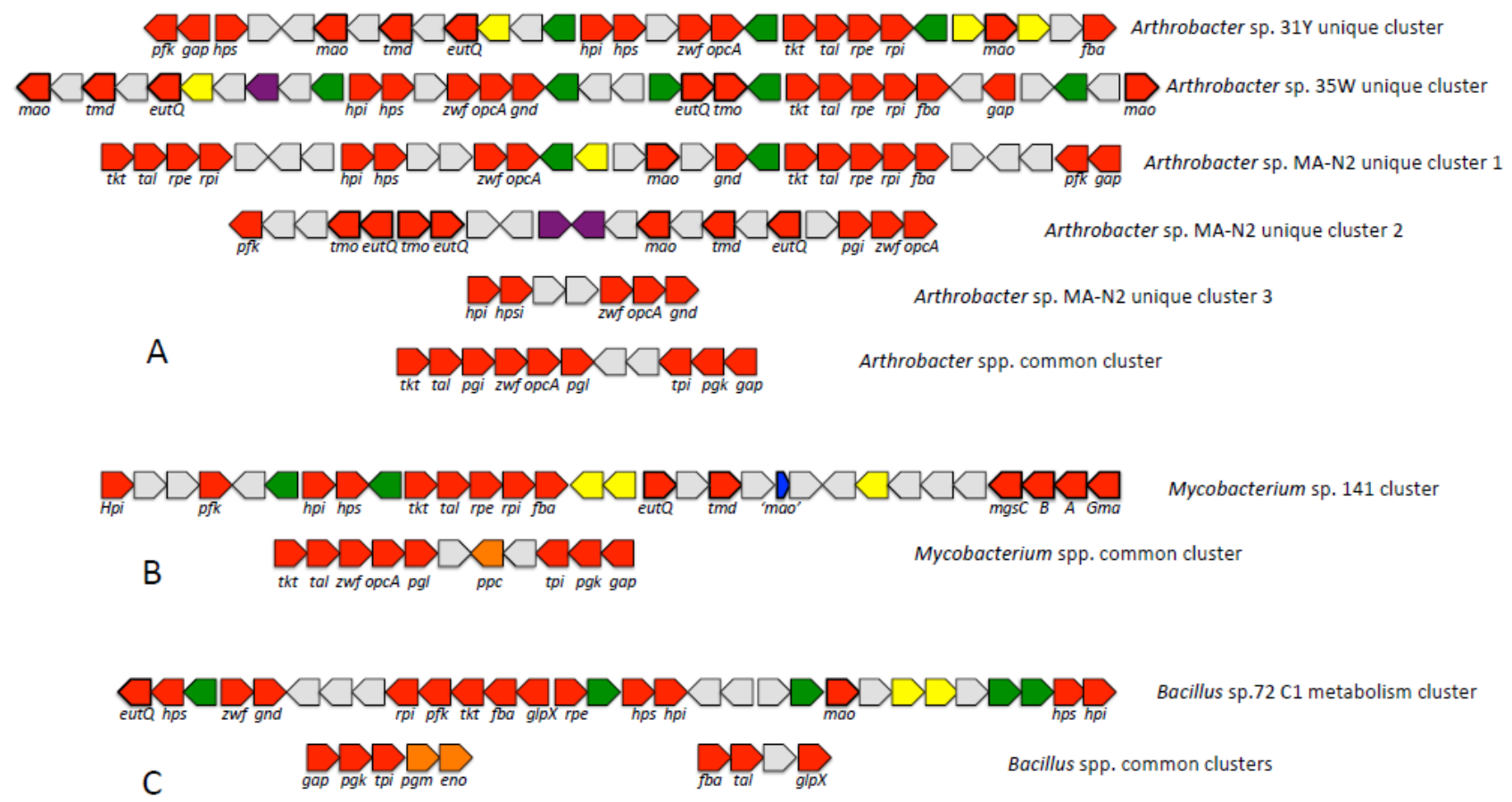

Figure 2. Methylotrophy gene clusters identified in the genomes described. Clusters identified in Mycobacterium sp. 155 and Bacillus sp. 37A are syntenic to the clusters shown for Mycobacterium sp. 141 and Bacillus sp. 72, respectively. In red, genes for methylotrophy functions. Genes for primary oxidation have bold lines. In green, genes encoding regulatory functions. In yellow, genes encoding transport functions. In grey, hypothetical genes or non-methylotrophy genes. In purple, genes encoding transposases. In orange, genes not essential for methylotrophy, but involved in other pathways sharing reactions with the RuMP cycle. In blue, the truncated Mao gene in the Mycobacterium methylotrophy clusters. Gene designations as indicated in the text. Common clusters (i.e., present in all strains of this genus) are identifiable not only in the strains described here, but also in multiple genomes of, respectively, Arthrobacter, Mycobacterium and Bacillus species. These are likely not methylotrophy specific. Genes in the unique clusters (i.e., unique to each strain) of Arthrobacter are typically highly related to the genes in the unique clusters of Mycobacterium (AI > 80\%), suggesting lateral transfers; genes in the common clusters are less related (AI 50\%-60\%, in agreement with AAI), suggesting vertical inheritance.

Remarkably, only small fragments of the mao gene are identifiable in the genomes of the Mycobacterium strains, as parts of conserved genome islands containing other methylotrophy genes (Figure 2). As the sizes of the truncated genes are very similar (129 and 123 nucleotides, respectively), it seems likely that these genes are remnants of a deletion event. The scenario in which two independent assemblies resulted in these truncated genes is less likely. If no functional Mao is encoded in the Mycobacterium genomes, how then is methylamine oxidized by these species? We hypothesize that it is oxidized via the $N$-methylglutamate pathway, which is widespread among diverse microbial taxa [34-36]. In the vicinity of the truncated mao genes, in each genome, we identified a cluster of genes similar to the genes encoding gamma-glutamylmethylamide synthetase (Gma) and the three 
subunits of $\mathrm{N}$-methylglutamate synthase, well-characterized in Proteobacteria (MgsA-C) [34,35]. No such gene clusters are present in other genomes included in this study. Gene clusters were also identified in the Mycobacterium genomes potentially responsible for the $N$-methylglutamate dehydrogenase function (MgdA-D; Table 2), also essential for the $N$-methylglutamate pathway. $m g d A-D$ gene homologs at approximately 50\% AI were identified in Arthrobacter strains $31 \mathrm{Y}$ and MA-N2, but not in the remaining genomes (Table 2).

The product of trimethylamine oxidation by the monooxygenase is trimethylamine $N$-oxide, which is cleaved into dimethylamine and formaldehyde by trimethylamine $N$-oxide demethylase (Tmd; [1,18]. While this enzyme has been partially purified from a number of sources, including methylotrophic bacteria [19,37], the sequence of the protein responsible for this activity remained unknown. Recently, it has been proposed that an aminomethyltransferase carries out this function in Alphaproteobacteria, in whose genomes the respective gene is co-located with the gene for Tmo [38]. In the genomes characterized here, we were unable to identify such a gene in the vicinity of the Tmo genes. Instead, we observed the presence of a conspicuous gene encoding a protein predicted to be a ferredoxin and a flavodoxin, possessing a NAD(P)-binding motif. These predicted properties match the properties of the characterized Tmd enzymes [1,19,37], suggesting this gene as a candidate for encoding the Tmd. Clearly, this proposed function requires experimental verification. Other conspicuous genes were present in the vicinity of putative methylated amine oxidation genes, annotated as ethanolamine utilization genes EutQ, whose function so far remains unknown. These encode small proteins of the cupin family. In each case, almost identical copies of EutQ are found near Tmo and Mao genes (Figure 2), and these are highly related ( $>80 \% \mathrm{AI}$ ) among the Arthrobacter and the Mycobacterium species. EutQ-like genes are also found in the $\mathrm{C} 1$ gene cluster in Bacillus, but they are not related to the genes in Arthrobacter or Mycobacterium. We hypothesize that the eutQ genes encode proteins important for methylated amine oxidation, thus we included these proteins in our gene inventory analysis (Table 2, Figure 2).

\subsubsection{Potential for Oxidation of Formaldehyde to $\mathrm{CO}_{2}$ via Linear Pathways}

Gram-positive methylotrophs have been suggested to utilize the cyclic pathway for formaldehyde oxidation to $\mathrm{CO}_{2}$, based on high activities of the respective enzymes [1,18]. However, experimental evidence was obtained for $B$. methanolicus that a linear pathway is also active [15]. We were able to identify genes for the relevant $\mathrm{H}_{4} \mathrm{~F}$-linked $\mathrm{C} 1$ transfers in all of the genomes. With the exception of strain $31 \mathrm{Y}$, a single FolD (bifunctional methylene- $\mathrm{H}_{4} \mathrm{~F}$ dehydrogenase/methenyl- $\mathrm{H}_{4} \mathrm{~F}$ cyclohydrolase) was encoded, and their reciprocal AI fit well within the AAI ranges (Figure 1). The genome of strain $31 \mathrm{Y}$ encoded a second FolD, with only 32\% AI with its homolog. The latter gene was part of a unique gene cluster encoding $\mathrm{C} 1$ transfer reactions, including an additional, non-homologous methenyl- $\mathrm{H}_{4} \mathrm{~F}$ cyclohydrolase (Mch), PurU (formyl- $\mathrm{H}_{4} \mathrm{~F}$ deformylase), a formaldehyde dehydrogenase (NAD-independent), an aminomethyltransferase and a molybdenum oxidoreductase of unknown function (genes 2855-2863). Only one other genome encoded a recognizable formaldehyde dehydrogenase (Fadh), the genome of Mycobacterium sp. 141, with 78\% AI to its counterpart in Arthrobacter sp. 31Y. Only the genomes of Arthrobacter strains $31 \mathrm{Y}$ and MA-N2 encoded formyl- $\mathrm{H}_{4} \mathrm{~F}$ ligases similar to the one in the genome of B. methanolicus (Fhs) [13,15]. However, all genomes 
encoded PurU enzymes: a single copy in the Bacillus genomes, two copies (similar, but not identical) in the Mycobacterium genomes and one to three relatively divergent copies in the Arthrobacter genomes. The prevalence of PurU (an irreversible enzyme) over Fhs (FtfL, a reversible enzyme) [39] indeed supports a proposal for the dissimilatory function of the $\mathrm{H}_{4} \mathrm{~F}-$-linked pathway [15].

Formate dehydrogenases $(\mathrm{Fdh})$ are encoded by all genomes, and they represent a variety of phylogenetically distant types. The genomes of the Mycobacterium species encode a three-subunit type (Table 2). Only one Arthrobacter strain, MA-N2, encodes a homolog of this enzyme (AI in the range of AAI). The genomes of the Bacillus species encode a single-subunit enzyme (Fdh2) highly similar to the one annotated in B. methanolicus [13,15], but without homologs in either Arthrobacter or Mycobacterium. The Arthrobacter species encode three other types unrelated to the first two, as follows. Strains 31Y and MA-N2 encode Fdh3 (AI 90\%); strains 31Y and 35W encode an Fdh4 (AI 73\%); and MA-N2 encodes Fdh5 that is equally distantly related to Fdh3 and Fdh4 (AI 52\%-58\%) and unrelated to Fdh1 and Fdh2.

\subsubsection{Oxidation of Formaldehyde to $\mathrm{CO}_{2}$ by the Cyclic Pathway}

Gram-positive methylotrophs are known to use the dissimilatory RuMP cycle for formaldehyde oxidation to $\mathrm{CO}_{2}[1,37]$. This cycle shares the early reactions, catalyzed by hexulose phosphate synthase (Hps) and hexulose phosphate isomerase (Hpi), that produce fructose 6-phosphate, the genes for which will be described below. Genes for glucose phosphate isomerase (Pgi) were identified in all genomes, with two nearly-identical copies present in the Mycobacterium strains. Interestingly, the proteins encoded by the Arthrobacter strains were unrelated to the mycobacterial counterparts, with AI $(<30 \%)$ being significantly below AAI. The Bacillus enzymes were unrelated to the counterparts from both Mycobacterium and Arthrobacter (AI <29\%).

Glucose 6-phosphate dehydrogenases (Zwf) are also represented by a variety of phylogenetic types, with multiple copies present in each genome (Table 1). The Actinobacteria share one type, with AI 70\% between Arthrobacter and Mycobacterium species (above AAI). The remaining types are specific to individual groups with low AI among the groups $(<40 \%)$. The genomes encoded one to two 6-phosphogluconolactonases. The Arthrobacter counterparts were only distantly related to the mycobacterial counterparts (AI 46\%-47\%), and none shared any identity with the Bacillus counterparts.

The phylogenetic landscape of the enzyme concluding the oxidative cycle, 6-phosphogluconate dehydrogenase (Gnd), was also complex, with three recognizable types being encoded. All three groups appeared to share one type, with AI values slightly above AAI. The second type was only shared among the Actinobacteria, again with AI being above AAI. The third type was exclusive to Bacillus.

\subsubsection{The Assimilatory RuMP Cycle}

Multiple copies of the first enzyme of the RuMP cycle, Hps, were identified in all genomes, in each case identical or nearly identical to each other within each genome. The actinobacterial genomes contained two copies each, and the counterparts from Mycobacterium and Arthrobacter revealed AI ( $87 \%$ to $91 \%$ ) that was significantly higher than AAI, suggesting that these enzymes were likely shared via lateral transfers among Actinobacteria. The Bacillus genomes each contained five copies of Hps genes, and these revealed low AI (41\%-43\%) with the actinobacterial counterparts, in agreement 
with AAI. One to three genes were identified in each genome encoding Hpi, and the trend for relative evolutionary distances was repeated: the actinobacterial counterparts were more related to each other than expected from AAI (74\%-77\%), while the Bacillus counterparts were related to the actinobacterial counterparts at the AAI value. Multiple phosphofructokinases are also encoded. While in the Arthrobacter species, most copies are highly related (the exception is gene 4492 in Arthrobacter 31Y), the Mycobacterium species encode two dissimilar enzymes. One shares unusually high AI (90\%) with Arthrobacter, suggesting a lateral transfer event, while the second is shared among the species at AI equaling AAI, suggesting vertical inheritance. Two distinct types are present in Bacillus (60\% AI). Multiple copies of fructose bisphosphate aldolases (Fba) were present, of which one was shared by Arthrobacter and Mycobacterium species at AI $>80 \%$, the other being more divergent between these species. The Bacillus counterparts were unrelated to the actinobacterial counterparts. Similar trends were observed for many of the enzymes that participate in regeneration of the acceptor molecule as part of the RuMP cycle [1]. Of the multiple copies of transketolase (Tkt) transaldolase (Tal), ribulose phosphate epimerase (Rpe) and ribulose phosphate isomerase (Rpi), fructose 1,6-sedoheptulose 1,7-bisphosphatase (GlpX), triosephosphate isomerase (Tpi), glyceraldehyde phosphate dehydrogenase (Gap) and phosphoglycerate kinase (Pgk), some copies revealed higher than expected AI between the Arthrobacter and Mycobacterium counterparts, while others were not phylogenetically related, suggesting complex histories.

To test for the potential for the alternative variant of the RuMP cycle, we queried the genomes for the presence of genes potentially encoding phosphogluconate dehydratase and 2-keto-3-deoxy6-phosphogluconate aldolase (Eda) [1]. Gene homologs for the latter were only detected in Arthrobacter strains $31 \mathrm{Y}$ and MA-N2, but gene homologs for the former were not detectable in any of the genomes.

\subsection{Analysis of Distinct C1 Gene Clusters Suggests a Means for the Evolution of Methylotrophy in Gram-Positive Methylotrophs}

In all of the genomes analyzed here, many of the genes implicated in methylotrophy were found as parts of genomic $\mathrm{C} 1$ metabolism islands (Figure 2; note that as these are draft genomes, the islands as shown may be incomplete, and some of the singleton genes may actually belong to islands). These islands in most cases contain genes for both primary $\mathrm{C} 1$ oxidation and the downstream functions that include oxidation and assimilation of formaldehyde. Clustering of $\mathrm{C} 1$ genes is also typical of other methylotrophs, including Proteobacteria, Verrucomicrobia and NC10 phylum species [39]. In cases where the regulation of gene or protein expression was studied, coordinated regulation was noted for genes/proteins involved in specific branches of $\mathrm{C} 1$ metabolic pathways [15,40], and co-transcription is a common mechanism [41]. Thus, one strategy for evolving methylotrophy seems to be clustering of relevant genes on the chromosomes. The other strategy must be multiplication of genes for key and likely rate-limiting functions, such as primary $\mathrm{C} 1$ oxidation and the early steps of $\mathrm{C} 1$ assimilation. Multiple and nearly identical copies were identified for these functions in most of the genomes analyzed here. Lateral transfers of gene clusters among diverse Gram-positive methylotrophs present another prominent mechanism. The proteins encoded by the specific $\mathrm{C} 1$ gene clusters in the Arthrobacter and the Mycobacterium species (Figure 2) display significantly higher relatedness than 
predicted from whole genome comparisons (Figure 1), strongly suggesting that these species either exchanged these clusters or acquired them from the same donor. However, an argument for the vertical evolution of methylotrophy in Gram-positive bacteria is also valid, as proteins in $\mathrm{C} 1$ pathways are only distantly related between the Bacillaceae and Actinobacteria, while the biochemical schemes are essentially identical.

How many of the Arthrobacter, Bacillus and Mycobacterium species are methylotrophic? Can we predict this capability from looking at the constantly growing genomic databases? Indeed, BLAST analyses identify homologs for most of the genes encoding functions necessary for methylotrophy (Table 2) in multiple sequenced genomes. However, no predictions could be made based on single genes. For example, even the "signature" functions, such as Hps and Hpi, are not necessarily indicative of methylotrophy, as they are also present in the genomes of non-methylotrophs $[38,42]$. The situation is further complicated by the fact that most of the reactions involved in assimilation or dissimilation of formaldehyde, including $\mathrm{C} 1$ transfer reactions or sugar phosphate inter-conversions, are common to all life. Thus, complete sets need to be identified in the genomes, with special attention to gene clustering. The genomes whose sequencing we are reporting here present some of the first blueprints for such analyses, in addition to the genomes of B. methanolicus [13-15].

\section{Conclusions}

The genomic sequences of seven novel strains representing the genera of Arthrobacter, Bacillus and Mycobacterium, all methylated amine utilizers, present an important reference dataset for understanding the genomic basis of methylotrophy in Gram-positive methylotrophic bacteria. This study is a major contribution to the field of methylotrophy, aimed at closing the gap in the genomic knowledge of $\mathrm{C} 1$ metabolism within this diverse group of bacteria.

\section{Acknowledgments}

This work was funded by grants from the National Science foundation (MCB-0950183) and the Department of Energy (DE-SC0010556) and was facilitated through the use of advanced computational storage and networking infrastructure provided by the Hyak supercomputer system supported in part by the University of Washington eScience Institute. The work conducted by the U.S. Department of Energy Joint Genome Institute was supported by the Office of Science of the U.S. Department of Energy under Contract No. DE-AC02-05CH11231.

\section{Author Contributions}

L.C., M.G.K. and M.E.L. conceived the project. M.G.K., T.L.M. and U.S. isolated pure cultures of organisms and isolated DNA. N.S. and T.W. supervised the JGI sequencing and analysis pipelines. L.C. and D.A.C.B. carried out comparative genome analysis. L.C. wrote the manuscript, all other authors contributed comments.

\section{Conflicts of Interest}

The authors declare no conflict of interest. 


\section{References}

1. Anthony, C. The Biochemistry of Methylotrophs; Academic Press: London, UK, 1982.

2. Chistoserdova, L.; Lidstrom, M.E. Aerobic methylotrophic prokaryotes. In The Prokaryotes, 4th ed.; Rosenberg, E., DeLong, E.F., Thompson, F., Lory, S., Stackebrandt, E., Eds.; Springer: Berlin Heidelberg, Germany, 2013; pp. 267-285.

3. Ward, N.; Larsen, Ø.; Sakwa, J.; Bruseth, L.; Khouri, H.; Durkin, A.S.; Dimitrov, G.; Jiang, L.; Scanlan, D.; Kang, K.H.; et al. Genomic insights into methanotrophy: The complete genome sequence of Methylococcus capsulatus (Bath). PLoS Biol. 2004, 2, e303.

4. Hou, S.; Makarova, K.S.; Saw, J.H.; Senin, P.; Ly, B.V.; Zhou, Z.; Ren, Y.; Wang, J.; Galperin, M.Y.; Omelchenko, M.V.; et al. Complete genome sequence of the extremely acidophilic methanotroph isolate V4, Methylacidiphilum infernorum, a representative of the bacterial phylum Verrucomicrobia. Biol. Direct 2008, 3, 26.

5. Khadem, A.F.; Wieczorek, A.S.; Pol, A.; Vuilleumier, S.; Harhangi, H.R.; Dunfield, P.F.; Kalyuzhnaya, M.G.; Murrell, J.C.; Francoijs, K.J.; Stunnenberg, H.G.; et al. Draft genome sequence of the volcano-inhabiting thermoacidophilic methanotroph Methylacidiphilum fumariolicum strain SolV. J. Bacteriol. 2012, 194, 3729-3730.

6. Ettwig, K.F.; Butler, M.K.; le Paslier, D.; Pelletier, E.; Mangenot, S.; Kuypers, M.M.; Schreiber, F.; Dutilh, B.E.; Zedelius, J.; de Beer, D.; et al. Nitrite-driven anaerobic methane oxidation by oxygenic bacteria. Nature 2010, 464, 543-548.

7. De Boer, L.; Brouwer, J.W.; van Hassel, C.W.; Levering, P.R.; Dijkhuizen, L. Nitrogen metabolism in the facultative methylotroph Arthrobacter P1 grown with various amines or ammonia as nitrogen sources. Antonie Van Leeuwenhoek 1989, 56, 221-232.

8. Vonck, J.; Arfman, N.; de Vries, G.E.; van Beeumen, J.; van Bruggen, E.F.; Dijkhuizen, L. Electron microscopic analysis and biochemical characterization of a novel methanol dehydrogenase from the thermotolerant Bacillus sp. C1. J. Biol. Chem. 1991, 266, 3949-3954.

9. De Vries, G.E.; Arfman, N.; Terpstra, P.; Dijkhuizen, L. Cloning, expression, and sequence analysis of the Bacillus methanolicus $\mathrm{C} 1$ methanol dehydrogenase gene. J. Bacteriol. 1992, 174, 5346-5353.

10. Zhang, X.; Fuller, J.H.; McIntire, W.S. Cloning, sequencing, expression, and regulation of the structural gene for the copper/topa quinone-containing methylamine oxidase from Arthrobacter strain P1, a Gram-positive facultative methylotroph. J. Bacteriol. 1993, 175, 5617-5627.

11. Bystrykh, L.V.; Vonck, J.; van Bruggen, E.F.; van Beeumen, J.; Samyn, B.; Govorukhina, N.I.; Arfman, N.; Duine, J.A.; Dijkhuizen, L. Electron microscopic analysis and structural characterization of novel NADP(H)-containing methanol: $N, N^{\prime}$-dimethyl-4-nitrosoaniline oxidoreductases from the Gram-positive methylotrophic bacteria Amycolatopsis methanolica and Mycobacterium gastri MB19. J. Bacteriol. 1993, 175, 1814-1822.

12. Park, H.; Lee, H.; Ro, Y.T.; Kim, Y.M. Identification and functional characterization of a gene for the methanol : $N, N^{\prime}$-dimethyl-4-nitrosoaniline oxidoreductase from Mycobacterium sp. strain JC1 (DSM 3803). Microbiology 2010, 156, 463-471. 
13. Heggeset, T.M.; Krog, A.; Balzer, S.; Wentzel, A.; Ellingsen, T.E.; Brautaset, T. Genome sequence of thermotolerant Bacillus methanolicus: features and regulation related to methylotrophy and production of L-lysine and L-glutamate from methanol. Appl. Environ. Microbiol. 2012, 78, 5170-5181.

14. Irla, M.; Neshat, A.; Winkler, A.; Albersmeier, A.; Heggeset, T.M.; Brautaset, T.; Kalinowski, J.; Wendisch, V.F.; Rückert, C. Complete genome sequence of Bacillus methanolicus MGA3, a thermotolerant amino acid producing methylotroph. J. Biotechnol. 2014, 188C, 110-111.

15. Müller, J.E.; Heggeset, T.M.; Wendisch, V.F.; Vorholt, J.A.; Brautaset, T. Methylotrophy in the thermophilic Bacillus methanolicus, basic insights and application for commodity production from methanol. Appl. Microbiol. Biotechnol. 2015, 99, 535-551.

16. Colby, J.; Zatman, L.J. Enzymological aspects of the pathways for trimethylamine oxidation and $\mathrm{C} 1$ assimilation of obligate methylotrophs and restricted facultative methylotrophs. Biochem. J. 1975, 148, 513-520.

17. Boulton, C.A.; Large, P.J. Inactivation of trimethylamine $N$-oxide aldolase (demethylase) during preparation of bacterial extracts. FEMS Microbiol. Lett. 1979, 5, 159-162.

18. Chen, Y.; Patel, N.A.; Crombie, A.; Scrivens, J.H.; Murrell, J.C. Bacterial flavin-containing monooxygenase is trimethylamine monooxygenase. Proc. Natl. Acad. Sci. USA 2011, 108, 17791-17796.

19. Kalyuzhnaya, M.G.; Lidstrom, M.E.; Chistoserdova, L. Utility of environmental probes targeting ancient enzymes: Methylotroph detection in Lake Washington. Microb. Ecol. 2004, 48, 436-472.

20. Beck, D.A.C.; McTaggart, T.L.; Setboonsarng, U.; Vorobev, A.; Kalyuzhnaya, M.G.; Ivanova, N.; Goodwin, L.; Woyke, T.; Lidstrom, M.E.; Chistoserdova, L. The expanded diversity of Methylophilaceae from Lake Washington through cultivation and genomic sequencing of novel ecotypes. PLoS One 2014, 9, e102458.

21. Beck, D.A.C.; McTaggart, T.L.; Setboonsarng, U.; Vorobev, A.; Kalyuzhnaya, M.G.; Goodwin, L.; Shapiro, N.; Woyke, T.; Lidstrom, M.E.; Chistoserdova, L. Multiphyletic origins of methylotrophy in Alphaproteobacteria, exemplified by comparative genomics of Lake Washington isolates. Environ. Microbiol. 2015, 17, 547-554.

22. Kalyuzhnaya, M.G.; Bowerman, S.; Lara, J.C.; Lidstrom, M.E.; Chistoserdova, L. Methylotenera mobilis gen. nov., sp. nov., an obligately methylamine-utilizing bacterium within the family Methylophilaceae. Int. J. Syst. Evol. Microbiol. 2006, 56, 2819-2823.

23. Chin, C.; Alexander, D.; Marks, P.; Klammer, A.; Drake, J.; Heiner, C.; Clum, A.; Copeland, A.; Huddleston, J.; Eichler, E.; et al. Nonhybrid, finished microbial genome assemblies from long-read SMRT sequencing data. Nat. Methods 2013, 10, 563-569.

24. Gnerre, S.; MacCallum, I.; Przybylski, D.; Ribeiro, F.J.; Burton, J.N.; Walker, B.J.; Sharpe, T.; Hall, G.; Shea, T.P.; Sykes, S.; et al. High-quality draft assemblies of mammalian genomes from massively parallel sequence data. Proc. Natl. Acad. Sci. USA 2011, 108, 1513-1518.

25. Zerbino, D.; Birney, E. Velvet: Algorithms for de novo short read assembly using de Bruijn graphs. Genome Res. 2008, 18, 821-829. 
26. Hyatt, D.; Chen, G.L.; Lacascio, P.F.; Land, M.L.; Larimer, F.W.; Hauser, L.J. Prodigal: Prokaryotic gene recognition and translation initiation site identification. BMC Bioinform. 2010, $11,119$.

27. Pati, A.; Ivanova, N.N.; Mikhailova, N.; Ovchinnikova, G.; Hooper, S.D.; Lykidis, A.; Kyrpides, N.C. GenePRIMP: A gene prediction improvement pipeline for prokaryotic genomes. Nat. Methods 2010, 7, 455-457.

28. Lowe, T.M.; Eddy, S.R. tRNAscan-SE: A program for improved detection of transfer RNA genes in genomic sequence. Nucl. Acids Res. 1997, 25, 955-964.

29. Pruesse, E.; Quast, C.; Knittel, K.; Fuchs, B.M.; Ludwig, W.; Peplies, J.; Glckner, F.O. SILVA: A comprehensive online resource for quality checked and aligned ribosomal RNA sequence data compatible with ARB. Nucl. Acids Res. 2007, 35, 7188-7196.

30. INFERNAL. Inference of RNA Alignments. Available online: http://infernal.janelia.org (accessed on 1 April 2014).

31. Markowitz, V.M.; Chen, I.M.; Palaniappan, K.; Chu, K.; Szeto, E.; Pillay, M.; Ratner, A.; Huang, J.; Woyke, T.; Huntemann, M.; et al. IMG 4 version of the integrated microbial genomes comparative analysis system. Nucl. Acids Res. 2014, 42, D560-D567.

32. Konstantinidis, K.T.; Tiedje, J.M. Towards a genome-based taxonomy for prokaryotes. J. Bacteriol. 2005, 187, 6258-6264.

33. Summers, M.L.; Wallis, J.G.; Campbell, E.L.; Meeks, J.C. Genetic evidence of a major role for glucose-6-phosphate dehydrogenase in nitrogen fixation and dark growth of the cyanobacterium Nostoc sp. strain ATCC 29133. J. Bacteriol. 1995, 177, 6184-6194.

34. Latypova, E.; Yang, S.; Wang, Y.S.; Wang, T.; Chavkin, T.A.; Hackett, M.; Schäfer, H.; Kalyuzhnaya, M.G. Genetics of the glutamate-mediated methylamine utilization pathway in the facultative methylotrophic beta-proteobacterium Methyloversatilis universalis FAM5. Mol. Microbiol. 2010, 75, 426-439.

35. Chen, Y.; Scanlan, J.; Song, L.; Crombie, A.; Rahman, M.T.; Schäfer, H.; Murrell, J.C. $\gamma$-glutamylmethylamide is an essential intermediate in the metabolism of methylamine by Methylocella silvestris. Appl. Environ. Microbiol. 2010, 76, 4530-4537.

36. Chen, Y.; McAleer, K.L.; Murrell, J.C. Monomethylamine as a nitrogen source for a nonmethylotrophic bacterium, Agrobacterium tumefaciens. Appl. Environ. Microbiol. 2010, 76, 4102-4104.

37. Parkin, K.L.; Hultinj, H.O. Characterization of trimethylamine- $N$-oxide (TMAO) demethylase activity from fish muscle microsomes. Biochemistry 1986, 100, 77-86.

38. Lidbury, I.; Murrell, J.C.; Chen, Y. Trimethylamine $N$-oxide metabolism by abundant marine heterotrophic bacteria. Proc. Natl. Acad. Sci. USA 2014, 111, 2710-2740.

39. Chistoserdova, L. Modularity of methylotrophy, revisited. Environ. Microbiol. 2011, 13, 2603-2622.

40. Peyraud, R.; Schneider, K.; Kiefer, P.; Massou, S.; Vorholt, J.A.; Portais, J.C. Genome-scale reconstruction and system level investigation of the metabolic network of Methylobacterium extorquens AM1. BMC Syst. Biol. 2011, 5, 189.

41. Kalyuzhnaya, M.G.; Lidstrom, M.E. QscR-mediated transcriptional activation of serine cycle genes in Methylobacterium extorquens AM1. J. Bacteriol. 2005, 187, 7511-7517. 
42. Kato, N.; Yurimoto, H.; Thauer, R.K. The physiological role of the ribulose monophosphate pathway in bacteria and archaea. Biosci. Biotechnol. Biochem. 2006, 70, 10-21.

(C) 2015 by the authors; licensee MDPI, Basel, Switzerland. This article is an open access article distributed under the terms and conditions of the Creative Commons Attribution license (http://creativecommons.org/licenses/by/4.0/). 